PROCEEDINGS OF THE

AMERICAN MATHEMATICAL SOCIETY

Volume 140, Number 8, August 2012, Pages 2575-2587

S 0002-9939(2011)11105-5

Article electronically published on November 28, 2011

\title{
BADLY APPROXIMABLE NUMBERS AND VECTORS IN CANTOR-LIKE SETS
}

\author{
S. G. DANI AND HEMANGI SHAH
}

(Communicated by Bryna Kra)

\begin{abstract}
We show that a large class of Cantor-like sets of $\mathbb{R}^{d}, d \geq 1$, contains uncountably many badly approximable numbers, respectively badly approximable vectors, when $d \geq 2$. An analogous result is also proved for subsets of $\mathbb{R}^{d}$ arising in the study of geodesic flows corresponding to $(d+1)$-dimensional manifolds of constant negative curvature and finite volume, generalizing the set of badly approximable numbers in $\mathbb{R}$. Furthermore, we describe a condition on sets, which is fulfilled by a large class, ensuring a large intersection with these Cantor-like sets.
\end{abstract}

\section{INTRODUCTION}

A real number $t$ is said to be badly approximable if there exists $\delta>0$ such that for every rational number $\frac{p}{q}$ we have $\left|t-\frac{p}{q}\right| \geq \frac{\delta}{q^{2}}$. Let $B$ denote the set of all badly approximable real numbers. It is well known that $B$ is a dense subset of $\mathbb{R}$ with Lebesgue measure 0. On the other hand, in [13] W.M. Schmidt showed, generalising a result of Jarnik, that not only $B$ has Hausdorff dimension 1, but so does $\bigcap_{i} f_{i}^{-1}(B)$ for any sequence $\left\{f_{i}\right\}$ of $C^{1}$ maps of $\mathbb{R}$ into itself with the derivatives not vanishing anywhere. This was achieved by studying the winning sets of a two-player infinite game that he introduced.

The ideas in [13] were later generalized by Schmidt 14 and by the first-named author $([5,6])$ to higher-dimensional situations. The techniques and results were applied in [5, and also later by other authors 1, to deduce certain geometric consequences concerning geodesics on manifolds of constant negative curvature and finite volume.

One may also ask whether given a compact totally disconnected subset of $\mathbb{R}$ we can conclude under some conditions that it must contain a badly approximable number. A similar question may also be asked about badly approximable vectors in compact subsets of $\mathbb{R}^{d}, d \geq 2$. For sets which are supports of a class of measures called "absolutely friendly measures", involving certain technical conditions that we shall not go into here, this is answered in the affirmative in [10], 8] and [9]. Further, for such sets $C$ it is shown that $B \cap C$ has Hausdorff dimension equal to that of $C$.

Received by the editors February 7, 2011 and, in revised form, March 3, 2011.

2010 Mathematics Subject Classification. Primary 11J25, 37D40, 37C35.

The second author thanks the Tata Institute of Fundamental Research, Mumbai, and the National Board for Higher Mathematics for support through Research Fellowships while this work was being done.

The authors thank the referee for helpful suggestions.

(C)2011 American Mathematical Society Reverts to public domain 28 years from publication 
In this paper we describe a class of subsets $\mathcal{L}$ of $\mathbb{R}^{d}$, and a class $\mathcal{C}$ of compact subsets of $\mathbb{R}^{d}$, such that for any $S \in \mathcal{L}$ and $C \in \mathcal{C}, S \cap C$ is uncountable. The argument is based on a generalisation of Schmidt's game. The conditions defining $\mathcal{L}$, signifying "largeness", are verified for the sets of badly approximable vectors, endpoint sets of geodesics in $\mathbb{R}^{d+1}$ with the hyperbolic metric whose image in a quotient with finite Riemannian volume is bounded (see $\S 3$ ), sets related to exceptional behaviour of orbits of automorphisms of toral quotients (see $\S 5$ ), etc. We are also able to relate the conditions to effects under transformations and countable intersections and prove the analogues of Schmidt's result mentioned above in the broader framework considered here.

The conditions for $\mathcal{C}$ involve certain simple regularity conditions, of admitting what we call a fine filter of coverings (FFC), and are in particular satisfied for Cantor-like sets and other fractal sets. Class $\mathcal{C}$ may be seen to be large from a heuristic point of view, and the conditions involved are flexible for applications, compared to seeking "absolutely friendly measures". In this context one may ask whether there exist compact subsets of $\mathbb{R}^{d}$ admitting FFC, the support of an absolutely friendly measure, and more specifically whether there exists a compact set admitting FFC, with Hausdorff dimension zero. At present answering these questions seems difficult. We note however that if the answers to the questions turn out to be in the negative, then the present approach would provide a simple and nontechnical method to conclude uncountability of the intersections, though without reference to Hausdorff dimension.

To describe the results we begin by introducing some definitions. We consider $\mathbb{R}^{d}, d \geq 1$, equipped with the usual Hilbert norm and the corresponding metric, denoted by $\|\cdot\|$ and $d(\cdot, \cdot)$ respectively. For subsets $S_{1}$ and $S_{2}$ of $\mathbb{R}^{d}, d\left(S_{1}, S_{2}\right)$ denotes $\inf \left\{\|x-y\| \mid x \in S_{1}, y \in S_{2}\right\}$; for $x \in \mathbb{R}^{d}$ and a subset $S, d(x, S)$ stands for $d(\{x\}, S)$. For a subset $S$ of $\mathbb{R}^{d}$ the thickness of $S$ is defined by

$$
\tau(S)=\inf _{V} \sup _{x, y \in S} d(x-y, V)
$$

where the infimum is taken over all hyperplanes $V$ in $\mathbb{R}^{d}$.

Definition 1.1. Let $C$ be a compact subset of $\mathbb{R}^{d}, d \geq 1$. By a filter of coverings of $C$ we mean a sequence $\mathcal{C}_{0}, \mathcal{C}_{1}, \mathcal{C}_{2}, \ldots$, where each $\mathcal{C}_{n}$ is a family of compact subsets covering $C$, such that the following conditions are satisfied:

(i) $\mathcal{C}_{0}=\{C\}$, and for $m \geq n \geq 0$ every $Y \in \mathcal{C}_{m}$ is contained in some $X$ in $\mathcal{C}_{n}$;

(ii) for all $n \geq 0$ every $X \in \mathcal{C}_{n}$ is covered by the sets $Y$ from $\mathcal{C}_{n+1}$ that are contained in $X$.

We shall only be concerned with filters of coverings satisfying certain regularity conditions as in the following definition.

Definition 1.2. Let $\left\{\mathcal{C}_{n}\right\}$ be a filter of coverings on a compact subset $C$ of $\mathbb{R}^{d}$. It is said to be a fine filter of coverings ( $F F C$ for short) if the following conditions are satisfied:

(i) there exist $\theta \in(0,1)$ and $a, b>0$ such that for any $n=0,1, \ldots$ and $X \in \mathcal{C}_{n}$, $a \theta^{n} \leq \operatorname{diam} X \leq b \theta^{n}$

(ii) there exists $\sigma \in\left(0, \frac{1}{2}\right)$ such that for any even integer $n \geq 0$ and $X \in \mathcal{C}_{n}$, if $Y \in \mathcal{C}_{n+1}$ and $Y \subset X$, then $\operatorname{diam}(Y) \leq \sigma \tau(X)$.

Remark 1.3. In the above definition one could consider keeping track of the $\sigma$ involved in (ii), introducing the notion of $\sigma$-fine, and even allowing $\sigma$ to be from 
the larger interval $(0,1)$, but since we will be concerned only with the condition in the above form, in the interest of simplicity we have chosen not to do so.

It is easy to see that the ternary Cantor set $C$ admits an FFC, with $\sigma=\frac{1}{3}$ : realizing $C$ as the set of real numbers in $[0,1]$ admitting a ternary expansion with only 0 and 2, for $n \in \mathbb{N}$ let $\mathcal{C}_{n}$ be the covering by sets consisting of all numbers with a given $n$-block for the first $n$ digits in the ternary expansion. One can also analogously construct compact totally disconnected subsets $C$ of $\mathbb{R}$ equipped with FFCs $\left\{\mathcal{C}_{n}\right\}$, where $\mathcal{C}_{n}$ consists of closed sets with widely nonuniform diameters (unlike in the Cantor set itself) that nevertheless satisfy the regularity conditions for FFC. Thus the class of compact sets admitting FFC is quite large, in $\mathbb{R}$ as well as in $\mathbb{R}^{d}, d \geq 2$; in particular various fractal sets such as the Sierpinski gasket, Sierpinski carpet, etc., can be seen to admit FFCs.

A class of examples of FFCs, related to Schmidt's $(\alpha, \beta)$-game can be defined on closed intervals $[a, b], a, b \in \mathbb{R}$, through which our technical results in the following sections correspond to Schmidt's results in the case of intervals (see $\S 2$ for details).

In the following sections we shall extend the idea of the Schmidt game, and winning sets, to compact sets with FFCs and prove various classes of sets to be winning sets. The results in particular yield the following consequences.

Theorem 1.4. Let $C$ be a compact subset of $\mathbb{R}$ admitting an FFC. For each $i \in \mathbb{N}$, let $\Omega_{i}$ be a neighbourhood of $C$ in $\mathbb{R}$ and $f_{i}: \Omega_{i} \rightarrow \mathbb{R}$ be a bi-Lipschitz map. Then there exist uncountably many $t$ in $C$ such that $f_{i}(t)$ is badly approximable for all $i$.

The set of badly approximable numbers generalises to a class of sets in $\mathbb{R}^{d}, d \geq 1$, involved in the study of geodesic flows on hyperbolic manifolds of finite volume (discussed earlier in [5]), and an analogous result holds for these more general sets (see Corollary 3.10).

We recall that a vector $v \in \mathbb{R}^{d}$ is said to be badly approximable if there exists $\delta>0$ such that for any $\mathbf{p} \in \mathbb{Z}^{d}$ and $q \in \mathbb{N}$

$$
\left\|v-\frac{\mathbf{p}}{q}\right\| \geq \delta q^{-(d+1) / d}
$$

For $d=1$ the condition corresponds to $v$ being a badly approximable number. For the set of badly approximable vectors in $\mathbb{R}^{d}, d \geq 2$, we prove the following.

Theorem 1.5. Let $C$ be a compact subset of $\mathbb{R}^{d}, d \geq 2$, admitting an FFC. Let $\left\{A_{i}\right\}$ be a sequence of affine automorphisms of $\mathbb{R}^{d}$ into itself. Then there exist uncountably many $v$ in $C$ such that $A_{i}(v)$ is badly approximable for all $i$.

As another application of our main result, in $\S 5$ we prove a strengthening of the main theorem of [7. For a continuous surjective endomorphism $\rho$ of $\mathbb{T}^{d}, d \geq 1$ and $x \in \mathbb{T}^{d}$, let $C_{\rho}$ denote the closure of the orbit of $x$ under $T$, namely $C_{\rho}(x)=$ $\overline{\left\{\rho^{i}(x) \mid i=0,1,2, \ldots\right\}}$. We realize $\mathbb{T}^{d}$ as $\mathbb{R}^{d} / \mathbb{Z}^{d}$ as usual and denote by $\pi: \mathbb{R}^{d} \rightarrow \mathbb{T}^{d}$ the canonical quotient map. Let $F$ be the subgroup of $\mathbb{T}^{d}$ consisting of all elements of finite order, namely $F=\pi\left(\mathbb{Q}^{d}\right)$, which is a dense subgroup of $\mathbb{T}^{d}$. Our results in particular yield the following conclusion:

Theorem 1.6. Let $C$ be a compact subset of $\mathbb{R}^{d}, d \geq 1$, admitting an FFC. Then there exists an uncountable subset $E$ of $C$ such that for any $v \in E$ and any surjective endomorphism $\rho$ of $\mathbb{T}^{d}, C_{\rho}(\pi(v)) \cap F=\emptyset$. 
For compact sets which are supports of absolutely friendly measures, the result is proved in [3, concluding also that the Hausdorff dimension of the intersection coincides with that of the set; their result concerns also avoiding a larger class of sets $F$ than those involved above.

\section{Games AND Winning SETS}

To each filter of coverings on a compact set $C$ we associate a two-player game as follows. Let $\mathcal{P}$ and $\mathcal{P}^{\prime}$ denote the players. A sample play begins with $\mathcal{P}$ picking a subset $S_{1}$ from $\mathcal{C}_{1}$. Then $\mathcal{P}^{\prime}$ picks a subset $S_{2}$ from $\mathcal{C}_{2}$, contained in $S_{1}$. This is followed by $\mathcal{P}$ picking a subset $S_{3}$ from $\mathcal{C}_{3}$ contained in $S_{2}$, and so on. Recursively after $\mathcal{P}^{\prime}$ has chosen a subset $S_{2 n}$ from $\mathcal{C}_{2 n}, \mathcal{P}$ chooses a subset $S_{2 n+1}$ from $\mathcal{C}_{2 n+1}$ contained in $S_{2 n}$ and then $\mathcal{P}^{\prime}$ picks a subset $S_{2 n+2}$ from $\mathcal{C}_{2 n+2}$ contained in $S_{2 n+1}$. As $\left\{S_{n}\right\}$ is a decreasing sequence of compact sets, the intersection of the sequence is nonempty. Winning the game is defined in terms of a preassigned set, say $W$. The player $\mathcal{P}$ is declared the winner if $\bigcap_{1}^{\infty} S_{n}$ is contained in $W$; otherwise $\mathcal{P}^{\prime}$ is the winner.

A (nonempty) subset $W$ of $C$ is called a winning set, with respect to $\left\{\mathcal{C}_{n}\right\}$, if there is a strategy by which $\mathcal{P}$ can ensure, by means of the choices during her turns, and irrespective of the choices made by $\mathcal{P}^{\prime}$, that $\bigcap_{1}^{\infty} S_{n}$ is contained in $W$.

Let $C$ be a compact subset of $\mathbb{R}^{d}, d \geq 1$, and let $\left\{\mathcal{C}_{n}\right\}$ be an FFC of $C$. Let $m \in \mathbb{N}$ and $\mathcal{C}^{(m)}=\left\{\mathcal{C}_{n}^{(m)}\right\}$ be the family of coverings of $C$ defined by $\mathcal{C}_{n}^{(m)}=\mathcal{C}_{2^{m} n}$ if $n$ is even and $\mathcal{C}_{2^{m}(n-1)+1}$ if $n$ is odd. We note that each $\left\{\mathcal{C}_{n}^{(m)}\right\}$ is an FFC of $C$, with the constants $\theta^{2^{m}}$ in place of $\theta, a$ as above and $b \theta^{1-2^{m}}$ in place of $b$. Clearly, a winning set with respect to $\left\{\mathcal{C}_{n}^{(m)}\right\}, m \geq 1$, is a winning set with respect to $\left\{\mathcal{C}_{n}\right\}$; during the turns which do not correspond to $\left\{\mathcal{C}_{n}^{(m)}\right\}$, the player $\mathcal{P}$ can make her choices arbitrarily within the stipulations of the game. We say that a subset $W$ of $C$ is a uniformly winning set if it is a winning set with respect to $\left\{\mathcal{C}_{n}^{(m)}\right\}$ for all $m$.

Lemma 2.1. For each $m \in \mathbb{N}$ let $W_{m}$ be a winning subset in $C$ with respect to $\left\{\mathcal{C}_{n}^{(m)}\right\}$. Then $\bigcap_{m=1}^{\infty} W_{m}$ is a winning set with respect to $\left\{\mathcal{C}_{n}\right\}$. In particular, the intersection of any sequence of uniformly winning sets is a uniformly winning set.

Proof. The proof is analogous to that of Theorem 2 in 13 . The strategy to be followed by $\mathcal{P}$, for choosing the set from $\mathcal{C}_{2 k+1}, k=0,1, \ldots$, given the choice made by $\mathcal{P}^{\prime}$ from $\mathcal{C}_{2 k}$ in the preceding turn shall be as follows. If $k$ is even, namely from $\mathcal{C}_{1}, \mathcal{C}_{5}, \mathcal{C}_{9}, \ldots$ which belong to $\mathcal{C}^{(1)}$, the choice is made of adopting the strategy to ensure the point of intersection of this sequence of sets to be contained in $W_{1}$. Along the sequence of odd $k$ such that $(k-1) / 2$ is even, or equivalently $k$ not congruent to $-1 \bmod 4$, the choice is made of adopting the strategy to ensure the point of intersection of the corresponding sets, coming from $\mathcal{C}^{(2)}$, to be contained in $W_{2}$. In general, along the sequence of $k$ which are congruent to -1 modulo $2^{l}$ for all $l<m$ and are not congruent to $-1 \bmod 2^{m}$, the sets are chosen so that the point of intersection of the corresponding sequence is contained in $W_{m}$. This describes a complete and consistent strategy such that the point of intersection lies in $\bigcap_{m=1}^{\infty} W_{m}$. This proves the lemma.

Lemma 2.2. Let $C$ be a compact subset and $\left\{\mathcal{C}_{n}\right\}$ be an $F F C$ on $C$. Then the complement of any countable subset of $C$ is a winning set with respect to $\left\{\mathcal{C}_{n}\right\}$. In particular every winning set is uncountable. 
Proof. Let $\left\{v_{i} \mid i \in \mathbb{N}\right\}$ be a given countable set. The player $\mathcal{P}$ shall choose $S_{2 k+1}$, $k=0,1, \ldots$ so that $v_{k}$ is not contained in $S_{2 k+1}$ for any $k$ : if $v_{k}$ is not contained in $S_{2 k}$, then there is nothing to be done, and if $v_{k} \in S_{2 k}$, then $\mathcal{P}$ shall choose $S_{2 k+1}$ to be an element of $\mathcal{C}_{2 k+1}$ containing a point of $S_{2 k}$ which is at a maximum possible distance from $v_{k}$; if $w$ is such a point, then $d\left(v_{k}, w\right) \geq \frac{1}{2} \operatorname{diam} S_{2 k}$, and since $\operatorname{diam} S_{2 k+1} \leq \sigma \operatorname{diam} S_{2 k}<\frac{1}{2} \operatorname{diam} S_{2 k}$, it follows that $v_{k}$ is not contained in $S_{2 k+1}$. This shows that $C \backslash\left\{v_{i}\right\}$ is a winning set. The second statement follows from the fact that if $W$ is a winning set, then by the first part and Lemma 2.1. $W \cap\left(C \backslash\left\{v_{i}\right\}\right)$ is a winning set and hence nonempty for any countable set $\left\{v_{i}\right\}$.

Remark 2.3. The game as above is related to the Schmidt game as follows. Let $B$ be a closed ball in $\mathbb{R}^{d}, d \geq 1$, with positive radius. Let $\alpha, \beta \in(0,1)$ be given. For each $n \in \mathbb{N}$ let $\mathcal{C}_{n}^{B}$ be the coverings of $B$ by closed balls of the form $\bar{B}\left(x, r_{n}\right)$ contained in $B$, where $r_{n}=\alpha^{(n-[n / 2])} \beta^{[n / 2]}([n / 2]$ denotes the largest integer not exceeding $n / 2$ ). Then $\left\{\mathcal{C}_{n}^{B}\right\}$ is seen to be a filter of coverings of $B$ and to be a fine filter, FFC, if $\alpha<\frac{1}{2}$. It may be seen that a subset $E$ of $\mathbb{R}^{d}$ is a winning set for the Schmidt game for $(\alpha, \beta)$ if and only if for every closed ball $B$ in $\mathbb{R}^{d}$, $E \cap B$ is a winning set for the game on $B$ associated to $\left\{\mathcal{C}_{n}^{B}\right\}$. The results in the following sections can be applied to deduce that various subsets of $\mathbb{R}^{d}$ are winning sets for Schmidt's $(\alpha, \beta)$-game. It may be noted however that we restrict ourselves to fine filters of coverings, and hence such an application may be made only with $\alpha<\frac{1}{2}$. Thus, for instance, we can conclude from Theorem 4.3 that the set of badly approximable vectors is a winning set for the $\operatorname{Schmidt}(\alpha, \beta)$-game when $\alpha<\frac{1}{2}$; the statement is known (see [14, Theorem 2A on page 53) for all pairs $(\alpha, \beta)$ such that $1-2 \alpha+\alpha \beta>0$, which on the other hand turns out also to be a necessary condition. With refinements in our methods it would be possible to cover the general assertion, which we have chosen not to undertake here in the interest of simplicity of proofs. Our focus here has been on extending the underlying theme to obtain results on intersections with more general compact sets, and the FFC condition seems to provide a natural framework for this purpose.

\section{A CLASS OF LARGE SETS}

In this section we introduce a condition on subsets of $\mathbb{R}^{d}$ and prove the main technical result about intersection of such sets with compact sets admitting FFC.

Definition 3.1. A subset $E$ of $\mathbb{R}^{d}$ is called an $\mathcal{L}$-set if given any $\lambda \in(0,1)$ there exists a family $\{S(p, t)\}$ of subsets of $\mathbb{R}^{d}$ (doubly) indexed over $p$ in a countable set $P$ and $t \in[0,1]$, and a family $\left\{\tau_{p}\right\}_{p \in P}$ of positive numbers bounded by 1 , such that the following holds:

i) $\tau(S(p, t)) \leq t \tau_{p} \leq 1$ for all $p \in P$;

ii) there exist $\rho \in(0,1)$ and $\epsilon>0$ such that if $k \geq 0$ and $N_{k}=\{p \in P \mid$ $\left.\lambda^{k+1}<\tau_{p} \leq \lambda^{k}\right\}$, then either $N_{k}$ is empty or for any compact subset $X$ with $\operatorname{diam} X \leq \rho \lambda^{k+1}$ there exists $q \in N_{k}$ such that $S(p, t) \cap X$ is contained in $S(q, t)$ for all $p \in N_{k}$ and $t \in(0, \epsilon)$;

iii) $\bigcup_{\delta>0}\left(\mathbb{R}^{d} \backslash \bigcup_{p \in P} S(p, \delta)\right)$ is contained in $E$.

The conditions in the definition, albeit technical, are designed to cover a variety of situations as will be seen in the following sections. A natural class of examples of $\mathcal{L}$-sets that can be explicitly constructed in quite a flexible manner is seen in the 
following lemma; here and in the sequel for $x \in \mathbb{R}^{d}$ and $r>0$, let $B(x, r)$ denote the open ball $\left\{y \in \mathbb{R}^{d} \mid\|x-y\|<r\right\}$.

Lemma 3.2. Let $\left\{x_{i}\right\}$ be a sequence of distinct points in $\mathbb{R}^{d}$ and $\left\{r_{i}\right\}$ be a bounded sequence of positive numbers, such that $\left\|x_{i}-x_{j}\right\| \geq \sqrt{r_{i} r_{j}}$ for any $i \neq j$, and let $E=\bigcup_{\delta>0}\left(\mathbb{R}^{d} \backslash \bigcup_{1}^{\infty} B\left(x_{p}, \delta r_{p}\right)\right)$. Then $E$ is an $\mathcal{L}$-set.

Proof. As the set $E$ remains unchanged if we replace $r_{i}$ by $c r_{i}$ for each $i$, for a fixed $0<c \leq 1$, we may assume without loss of generality that $r_{i} \leq 1$ for all $i$. Let $\lambda \in$ $(0,1)$ be given. Let $P=\mathbb{N}$ and for all $p \in P$ and $0 \leq t \leq 1$, put $S(p, t)=B\left(x_{p}, t r_{p}\right)$ (independent of $\lambda$ ) and $\tau_{p}=r_{p}$ for all $p \in P$. Then $\tau(S(p, t))=t r_{p}=t \tau_{p} \leq 1$, for all $p$, so condition (i) in the definition holds. Now let $\rho \in(0,1)$ be arbitrary and let $\epsilon=\frac{1}{3}(1-\rho) \lambda$. Let $k \geq 0$ and let $N_{k}=\left\{p \in \mathbb{N} \mid \lambda^{k+1}<\tau_{p} \leq \lambda^{k}\right\}$. Then for any $p, q \in N_{k}$ and $t \in(0, \epsilon)$ we have $d\left(B\left(x_{p}, t r_{p}\right), B\left(x_{q}, t r_{q}\right)\right)=\left\|x_{p}-x_{q}\right\|-t\left(r_{p}+r_{q}\right) \geq$ $\sqrt{r_{p} r_{q}}-t\left(r_{p}+r_{q}\right)>\lambda^{k+1}-2 \epsilon \lambda^{k}>\rho \lambda^{k+1}$, as $\epsilon<\frac{1}{2}(1-\rho) \lambda$, and hence a compact subset $X$ of diameter at most $\rho \lambda^{k+1}$ intersects $B\left(x_{p}, t r_{p}\right)$ for at most one $p \in N_{k}$. This shows that condition (ii) holds. Hence $E$ is an $\mathcal{L}$-set.

Definition 3.3. We call a subset $E$ of $\mathbb{R}^{d}, d \geq 1$, a $\mathcal{B}$-set if $E$ is of the form $\bigcup_{\delta>0}\left(\mathbb{R}^{d} \backslash \bigcup_{i=1}^{\infty} B\left(x_{i}, \delta r_{i}\right)\right)$, where $\left\{x_{i}\right\}$ is a sequence in $\mathbb{R}^{d}$ and $\left\{r_{i}\right\}$ is a bounded sequence of positive numbers such that $\left\|x_{i}-x_{j}\right\| \geq \sqrt{r_{i} r_{j}}$ for all $i, j, i \neq j$.

Lemma 3.2 shows that every $\mathcal{B}$ set is an $\mathcal{L}$-set.

Remarks 3.4 .

(i) The set of badly approximable numbers is a $\mathcal{B}$-set (and hence an $\mathcal{L}$-set by Lemma 3.2); the sequence $\left\{x_{i}\right\}$ is chosen to be an enumeration of the rationals and if $x_{i}$ is the rational $p / q$ in reduced form, we choose $r_{i}=1 / q^{2}$.

(ii) Given a sequence $\left\{x_{i}\right\}$ in $\mathbb{R}^{d}, d \geq 1$, we can construct recursively a bounded sequence $\left\{r_{i}\right\}$ of positive numbers such that $\left\|x_{i}-x_{j}\right\| \geq \sqrt{r_{i} r_{j}}$ for any $i \neq j$, and conversely given a bounded sequence $\left\{r_{i}\right\}$ of positive numbers we can construct a sequence $\left\{x_{i}\right\}$ such that $\left\|x_{i}-x_{j}\right\| \geq \sqrt{r_{i} r_{j}}$ for all $i \neq j$.

(iii) If $E$ is a subset of $\mathbb{R}^{d}$ such that $\mathbb{R}^{d} \backslash E$ is countable, then $E$ is an $\mathcal{L}$-set: clearly, by (ii), $E$ contains a $\mathcal{B}$-set which by Lemma 3.2 is an $\mathcal{L}$-set, and hence $E$ is itself an $\mathcal{L}$-set.

(iv) If $\varphi: \mathbb{R}^{a} \rightarrow \mathbb{R}^{b}$, where $a, b \in \mathbb{N}$, is a surjective linear map and $E$ is an $\mathcal{L}$-set in $\mathbb{R}^{b}$, then $\varphi^{-1}(E)$ is an $\mathcal{L}$-set in $\mathbb{R}^{a}$.

Let $\Omega$ be a subset of $\mathbb{R}^{d}$ and $f: \Omega \rightarrow \mathbb{R}^{d}$ be a map of $\Omega$ into $\mathbb{R}^{d}$. We say that $f$ is bi-Lipschitz if there exists $c>0$ such that $c^{-1}\|u-v\| \leq\|f(u)-f(v)\| \leq c\|u-v\|$ for all $u, v \in \Omega$. We note the following.

Lemma 3.5. Let $C$ be a compact subset of $\mathbb{R}^{d}, \Omega$ a neighbourhood of $C$ and $f$ : $\Omega \rightarrow \mathbb{R}^{d}$ be a bi-Lipschitz map. Let $E$ be a $\mathcal{B}$-set in $\mathbb{R}^{d}$. Then there exists a $\mathcal{B}$-set $F$ of $\mathbb{R}^{d}$ such that $f^{-1}(E) \cap C=F \cap C$.

Proof. Let $\left\{x_{i}\right\}$ and $\left\{r_{i}\right\}$ be as in the definition of $E$ being a $\mathcal{B}$-set. Let $c>0$ be such that $c^{-1}\|u-v\| \leq\|f(u)-f(v)\| \leq c\|u-v\|$ for all $u, v \in \Omega$. Let $P=\{i \in$ $\left.\mathbb{N} \mid x_{i} \in f(\Omega)\right\}$. For $i \in P$ let $y_{i}=f^{-1}\left(x_{i}\right)$ (note that $f$ induces a homeomorphism of $\Omega$ and $f(\Omega)$ ). Let $F=\bigcup_{\delta>0} \mathbb{R}^{d} \backslash \bigcup_{i \in P} B\left(y_{i}, \delta r_{i}\right)$. We note that for $i \in P$, $B\left(y_{i}, \delta r_{i}\right)$ is contained in $f^{-1}\left(B\left(x_{i}, c \delta r_{i}\right)\right)$. It follows that $F$ contains $f^{-1}(E)$. Let $\delta_{0}=d\left(f(C), \mathbb{R}^{d} \backslash f(\Omega)\right)$. Then for $\delta<\delta_{0}$ and $i \notin P, C \cap f^{-1}\left(B\left(x_{i}, \delta r_{i}\right)\right)=\emptyset$. 
Also for $i \in P, B\left(y_{i}, \delta r_{i}\right)$ contains $f^{-1}\left(B\left(x_{i}, c^{-1} \delta r_{i}\right)\right)$. This shows that $F \cap C$ is contained in $f^{-1}(E)$. Thus $f^{-1}(E) \cap C=F \cap C$.

We now state and prove our main technical result; it is a generalisation of the main theorem of [5].

Theorem 3.6. Let $E$ be an $\mathcal{L}$-set in $\mathbb{R}^{d}$. Let $C$ be a compact subset of $\mathbb{R}^{d}$ and $\left\{\mathcal{C}_{n}\right\}$ be an FFC on $C$. Then $E \cap C$ is a uniformly winning set with respect to $\left\{\mathcal{C}_{n}\right\}$.

Proof. We note that in view of Lemma2.1 it suffices to show that $E \cap C$ is a winning set with respect to $\left\{\mathcal{C}_{n}\right\}$, as analogously we also get that $E \cap C$ is a winning set with respect to the $\left\{\mathcal{C}_{n}^{(m)}\right\}$, for all $m \in \mathbb{N}$. Now let $\theta, a, b$ and $\sigma$ be the constants as in the definition of $\left\{\mathcal{C}_{n}\right\}$ being an FFC. Let $E$ be a given $\mathcal{L}$-set. Choose $\lambda=\theta^{2}$ and let $\{S(p, t)\},\left\{\tau_{p}\right\}$, and $\rho \in(0,1)$ and $\epsilon>0$ be such that the conditions in the definition of $E$ being an $\mathcal{L}$-set hold. Let $k_{0} \in \mathbb{Z}^{+}$be such that $b \lambda^{k_{0}}<\rho$. Let $\delta<\min \left\{\left(\frac{1}{2 \sigma}-1\right) a \theta^{2 k_{0}+1}, \epsilon\right\}$ and $B_{\delta}=\mathbb{R}^{d} \backslash \bigcup_{p \in P} S(p, \delta)$. We shall show that there is a strategy by which $\mathcal{P}$ can ensure the point of intersection to be contained in $B_{\delta}$.

For any affine hyperplane $V$ in $\mathbb{R}^{d}$ and $\omega>0$ let $V(\omega)$ denote the open neighbourhood $\left\{u \in \mathbb{R}^{d} \mid d(u, V)<\omega\right\}$ of $V$.

The strategy to be adopted by $\mathcal{P}$ is as follows. Let $k \geq 0$ and let $S_{2 k}$ be the element of $\mathcal{C}_{2 k}$ chosen by $\mathcal{P}^{\prime} ; S_{2 k}=C$ if $k=0$. If $k<k_{0}$, then $\mathcal{P}$ may pick $S_{2 k+1}$ to be any element of $\mathcal{C}_{2 k+1}$ contained in $S_{2 k}$. Now suppose $k \geq k_{0}$ and let $N_{k}=\left\{p \in P \mid \theta^{2 k+2}<\tau_{p} \leq \theta^{2 k}\right\}$. If $N_{k}$ is empty, then $\mathcal{P}$ may choose $S_{2 k+1}$ to be any element of $\mathcal{C}_{2 k+1}$ contained in $S_{2 k}$. Next suppose that $N_{k}$ is nonempty. We note that $\operatorname{diam} S_{2 k} \leq b \theta^{2 k}<\rho \lambda^{k-k_{0}+1}$, and hence there exists $q \in N_{k-k_{0}}$ such that $S(p, t) \cap S_{2 k}$ is contained in $S(q, t)$ for all $p \in N_{k-k_{0}}$ and $t \in(0, \epsilon)$. Since $\tau(S(q, \delta)) \leq \delta \tau_{q} \leq \delta \lambda^{k-k_{0}}$ there exists an affine hyperplane $V_{k}$ such that $S(q, \delta)$ is contained in $V_{k}\left(\delta \lambda^{k-k_{0}}\right)$; hence $S(p, \delta) \cap S_{2 k}$ is contained in $V_{k}\left(\delta \lambda^{k-k_{0}}\right)$ for all $p \in N_{k-k_{0}}$. Let $x$ be the point of $S_{2 k}$ farthest from $V_{k}$; that is, $d\left(x, V_{k}\right) \geq d\left(y, V_{k}\right)$ for all $y \in C$. Then clearly $d\left(x, V_{k}\right) \geq \frac{1}{2} \tau\left(S_{2 k}\right)$, and by the condition of $\left\{\mathcal{C}_{n}\right\}$ being an FFC with the data as above, the latter is at least $\frac{1}{2 \sigma} \operatorname{diam} S_{2 k+1}$. Now $\mathcal{P}$ shall pick $S_{2 k+1} \in \mathcal{C}_{2 k+1}$ containing $x$. We note that $S_{2 k+1}$ is outside $V_{k}\left(\delta \lambda^{k-k_{0}}\right)$, since

$$
d\left(x, V_{k}\right)-\operatorname{diam} S_{2 k+1} \geq\left(\frac{1}{2 \sigma}-1\right) \operatorname{diam} S_{2 k+1} \geq\left(\frac{1}{2 \sigma}-1\right) a \theta^{2 k+1}>\delta \lambda^{k-k_{0}},
$$

in view of condition (ii) in the definition of FFC for $S_{2 k+1} \in \mathcal{C}_{1}$, and the choice of $\delta$. Therefore $S_{2 k+1}$ is disjoint from $S(p, \delta)$ for all $p \in N_{k}$. As this holds for all $k$, we get that the point of intersection of the sequence $\left\{S_{n}\right\}$ is contained in $B_{\delta}$. This proves the theorem.

We note that the image of an $\mathcal{L}$-set in $\mathbb{R}^{d}$ under any affine automorphism of $\mathbb{R}^{d}$ is again an $\mathcal{L}$-set. Hence together with Lemma 2.1 the theorem implies the following.

Corollary 3.7. Let $C$ be a compact set in $\mathbb{R}^{d}, d \geq 1$, equipped with an $F F C\left\{\mathcal{C}_{n}\right\}$. For each $i \in \mathbb{N}$ let $A_{i}$ be an affine automorphism of $\mathbb{R}^{d}$. Then for any $\mathcal{L}$-set $E$ in $\mathbb{R}^{d}, \bigcap_{i=1}^{\infty} A_{i}^{-1}(E) \cap C$ is a uniformly winning set in $C$ with respect to $\left\{\mathcal{C}_{n}\right\}$.

For $\mathcal{B}$-sets we can get the following stronger assertion.

Corollary 3.8. Let $C$ be a compact subset of $\mathbb{R}^{d}$ admitting an $F F C\left\{\mathcal{C}_{n}\right\}$. For each $i \in \mathbb{N}$, let $\Omega_{i}$ be a neighbourhood of $C$ in $\mathbb{R}^{d}$ and $f_{i}: \Omega_{i} \rightarrow \mathbb{R}^{d}$ be a bi-Lipschitz map. Let $E$ be a $\mathcal{B}$-set. Then $\bigcap_{i=1}^{\infty} f_{i}^{-1}(E) \cap C$ is a uniformly winning set with respect to $\left\{\mathcal{C}_{n}\right\}$. 
Proof. By Lemma 3.5 there exist $\mathcal{B}$-sets $F_{i}, i \in \mathbb{N}$, such that $f_{i}^{-1}(E) \cap C=F_{i} \cap C$. By Lemma 3.2 and Theorem 3.6. each $F_{i} \cap C$ is a winning set with respect to $\left\{\mathcal{C}_{n}^{(m)}\right\}$ for every $m$ and thus a uniformly winning set. Hence by Lemma $2.1 \bigcap_{i=1}^{\infty} F_{i} \cap C=$ $\bigcap_{i=1}^{\infty} f_{i}^{-1}(E) \cap C$ is a uniformly winning set with respect to $\left\{\mathcal{C}_{n}\right\}$.

Proof of Theorem 1.4. Let $C$ be a compact subset of $\mathbb{R}$ admitting FFC $\left\{\mathcal{C}_{n}\right\}$, and for $i \in \mathbb{N}$ let $\Omega_{i}$ and $f_{i}$ be as in the hypothesis of the theorem. Let $B$ denote the set of badly approximable numbers in $\mathbb{R}$. As $B$ is a $\mathcal{B}$-set (see Remark 3.4(i)) by Corollary 3.8 we get that $\bigcap_{i=1}^{\infty} f_{i}^{-1}(B) \cap C$ is a winning set with respect to $\left\{\mathcal{C}_{n}\right\}$. Hence by Lemma 2.2 $\bigcap_{i=1}^{\infty} f_{i}^{-1}(B) \cap C$ is uncountable. This proves the theorem.

Remark 3.9. It may be noted that by the regularity of the Lebesgue measure, there exist compact subsets of positive Lebesgue measure not containing any badly approximable number. Thus the conclusion as in Theorem 1.4 cannot be expected to hold for arbitrary compact subsets of $\mathbb{R}$. It may also be noted on the other hand that the sets above for which the conclusion of Theorem 1.4 does not hold provide examples of sets not admitting an FFC.

The instance of badly approximable numbers may be realised as a special case of a large class of geometric examples. We view $\mathbb{R}$ as the boundary of the upper half-plane $\mathbb{H}^{2}=\{x+i y \mid y>0\}$, equipped with the Poincaré metric. We recall that geodesics in $\mathbb{H}^{2}$ are either vertical lines or semicircles orthogonal to the real axis. The special linear group $S L(2, \mathbb{R})$ acts by isometries on $\mathbb{H}^{2}$, by $z \mapsto \frac{a z+b}{c z+d}$ under $\left(\begin{array}{ll}a & b \\ c & d\end{array}\right) \in S L(2, \mathbb{R})$. Given a point $p \in \mathbb{H}^{2}$ and $s \in \mathbb{R}$ there exists a unique (one-sided) geodesic $\left\{\gamma_{t}\right\}_{t=0}^{\infty}$ in $\mathbb{H}^{2}$, a segment of a Euclidean circle, tending to $s$ as $t \rightarrow \infty$, with $\gamma_{0}=p$; we call $s$ the endpoint of $\left\{\gamma_{t}\right\}_{t=0}^{\infty}$. The badly approximable numbers in $\mathbb{R}$ are precisely the endpoints of the geodesics $\left\{\gamma_{t}\right\}_{t=0}^{\infty}$ whose image modulo $S L(2, \mathbb{Z})$, namely in the quotient of $\mathbb{H}^{2}$ under the action of $S L(2, \mathbb{Z})$, is bounded (relatively compact). (The statement is independent of the chosen base point $p$ as above, since given two positive time geodesics with the same endpoint, if the image of one of them is bounded, then so is that of the other.)

More generally, given a discrete subgroup of $\Gamma$ of $S L(2, \mathbb{R})$ whose action on $\mathbb{H}^{2}$ has a fundamental domain which is noncompact but has finite hyperbolic area (viz. a nonuniform lattice in $S L(2, \mathbb{R})$; see 2] for generalities on this part) the set, say $E$, of points of $\mathbb{R}$ which are endpoints of the geodesics $\left\{\gamma_{t}\right\}_{t=0}^{\infty}$ whose image in the quotient surface $\Gamma \backslash \mathbb{H}^{2}$ is bounded is a $\mathcal{B}$-set (see [5], 6]; a more detailed exposition may be found in [4]). These geometrical analogues extend also to higher dimensions: $\mathbb{R}^{d}$ may be viewed canonically as the boundary of the $(d+1)$-dimensional hyperbolic space $\mathbb{H}^{d+1}$, and if $\Gamma$ is a discrete group of isometries of $\mathbb{H}^{d+1}$ whose action has a fundamental domain with finite volume (which may not be compact), then the set $E$ of points in $\mathbb{R}^{d}$ which are endpoints of geodesics in $\mathbb{H}^{d+1}$ whose image in $\Gamma \backslash \mathbb{H}^{d+1}$ is bounded is a $\mathcal{B}$-set (see [5], 6]). Thus we have

Corollary 3.10. Let $\Gamma$ be a discrete group of isometries of $\mathbb{H}^{d+1}$ whose action on $\mathbb{H}^{d+1}$ has a fundamental domain with finite volume. Let $p \in \mathbb{H}^{d+1}$ and $E$ be a set of points in $\mathbb{R}^{d}$ which are endpoints of geodesics $\left\{\gamma_{t}\right\}_{t=0}^{\infty}$ in $\mathbb{H}^{d+1}$ such that $\gamma_{0}=p$, and the image of $\left\{\gamma_{t}\right\}_{t \geq 0}$ in $\Gamma \backslash \mathbb{H}^{d+1}$ is bounded (has compact closure). Then for every 
compact subset of $\mathbb{R}^{d}$ equipped with an $F F C\left\{\mathcal{C}_{n}\right\}, E \cap C$ is a uniformly winning set in $C$.

As in the case of badly approximable numbers we can also take inverse images under bi-Lipschitz maps defined on neighbourhoods of $C$ and take countable intersections of pre-images and conclude that they are uncountable. We omit the details. In 12 it is shown that the set $E$ as above is "absolutely winning". This implies in particular that its image under any quasisymmetric homeomorphism of $\mathbb{R}^{d}$ is a winning set and hence intersects every nonempty open set in a set of Hausdorff dimension $d$. One may ask about the intersection of such images with compact totally disconnected sets as in Corollary 3.10. A similar comment applies also with regard to Theorem 1.4. The reader is also referred to [11] for a general result in the framework of flows on finite-volume homogeneous spaces induced by diagonalizable one-parameter subgroups, which in particular shows the set $E$ as above to be a winning set for certain modified Schmidt games.

\section{BADLY APPROXIMABLE VECTORS}

In this section we show that compact subsets of $\mathbb{R}^{d}$ equipped with an FFC, contain uncountably many badly approximable vectors. For this we shall prove the following.

Theorem 4.1. For all $d \geq 1$ the set of all badly approximable vectors in $\mathbb{R}^{d}$ is an $\mathcal{L}$-set in $\mathbb{R}^{d}$.

We first note the following lemma; the argument is along the lines of the proof of Theorem 2A on page 53 in [14, which deals with the two-dimensional case.

Lemma 4.2. Let $\lambda, \rho \in(0,1)$ be given constants and let $\delta=\frac{1}{3}(1-\rho) \lambda$. Let $k \in \mathbb{N}$ be arbitrary. Let $X$ be a compact subset of $\mathbb{R}^{d}$ with diameter at most $\rho \lambda^{k+1}$. Let $Q$ be the set of rationals $\frac{\mathbf{p}}{q} \in \mathbb{R}^{d}$, with $\mathbf{p} \in \mathbb{Z}^{d}$ and $q \in \mathbb{N}$, such that $\lambda^{k+1}<q^{-(d+1) / d} \leq$ $\lambda^{k}$ and $X \cap B\left(\frac{\mathbf{p}}{q}, \delta q^{-d+1 / d}\right) \neq \emptyset$. Then $Q$ is contained in an affine hyperplane in $\mathbb{R}^{d}$.

Proof. We note that if $x=\frac{\mathbf{p}}{q}, x^{\prime}=\frac{\mathbf{p}^{\prime}}{q^{\prime}} \in Q$, then

$$
\left\|x-x^{\prime}\right\|<\delta\left(q^{-(d+1) / d}+q^{\prime-(d+1) / d}\right)+\operatorname{diam} X \leq 2 \delta \lambda^{k}+\rho \lambda^{k+1}<\lambda^{k+1} .
$$

Suppose that $Q$ is not contained in any hyperplane. Then there exist $d+1$ points, say $x_{1}, x_{2}, \ldots, x_{d+1}$ in $Q$ which are not contained in any affine hyperplane in $\mathbb{R}^{d}$. Let $\Delta=\Delta\left(x_{1}, x_{2}, \ldots, x_{d+1}\right)$ denote the $d$-dimensional simplex subtended by $x_{1}, x_{2}, \ldots, x_{d+1}$. The volume of the simplex satisfies the relation

$$
V(\Delta) \leq(d !)^{-1}\left\|x_{1}-x_{2}\right\|\left\|x_{1}-x_{3}\right\| \ldots\left\|x_{1}-x_{d+1}\right\| .
$$

Hence by the estimates as above we have $V(\Delta)<(d !)^{-1} \lambda^{(k+1) d}$. On the other hand, $V(\Delta)=(d !)^{-1}|\operatorname{det} L|$, where

$$
L=\left(\begin{array}{ccccc}
1 & x_{11} & x_{12} & \ldots & x_{1 d} \\
1 & x_{21} & x_{22} & \ldots & x_{2 d} \\
\vdots & \vdots & \vdots & & \vdots \\
1 & x_{d 1} & x_{d 2} & \ldots & x_{d d} \\
1 & x_{(d+1) 1} & x_{(d+1) 2} & \ldots & x_{(d+1) d}
\end{array}\right)
$$


where $x_{i j}, j=1, \ldots, d$, denotes the $j$ th coordinate of $x_{i}$, for all $i=1, \ldots, d+1$. Then det $L$ is a nonzero rational number with denominator a divisor of $q_{1} q_{2} \ldots q_{d+1}$, and hence in particular $|\operatorname{det} L| \geq\left|q_{1} q_{2} \cdots q_{d+1}\right|^{-1}$. As $x_{i} \in Q,\left|q_{i}\right|^{-1}>\left(\lambda^{k+1}\right)^{d / d+1}$ for all $i$, and hence we get that $|\operatorname{det} L|>\lambda^{(k+1) d}$. In turn this implies that $V(\Delta)>(d !)^{-1} \lambda^{(k+1) d}$, which is a contradiction. Hence $Q$ is contained in an affine hyperplane.

Proof of Theorem 4.1. Let $\lambda \in(0,1)$ be given. Let $P$ be the set of pairs $(V, k)$, where $V$ is a proper rational affine subspace of $\mathbb{R}^{d}$ and $k \in \mathbb{Z}^{+}$. For each $k \in \mathbb{Z}^{+}$let $M_{k}=\left\{q \in \mathbb{N} \mid \lambda^{k+1}<q^{-(d+1) / d} \leq \lambda^{k}\right\}$. For $p=(V, k) \in P$ and $t \in[0,1]$ let $S(p, t)$ be the set of vectors $v$ in $\mathbb{R}^{d}$ for which there exist $\mathbf{p} \in \mathbb{Z}^{d}$ and $q \in M_{k}$ such that $\underline{\mathbf{p}} \in V$ and $\left\|v-\frac{\mathbf{p}}{q}\right\|<t q^{-(d+1) / d}$. Then $\bigcup_{\delta>0}\left(\mathbb{R}^{d} \backslash \bigcup_{p \in P} S(p, \delta)\right)$ is precisely the set of badly approximate vectors in $\mathbb{R}^{d}$. For $p=(V, k) \in P$, let $\tau_{p}=\sup _{q \in M_{k}} q^{-(d+1) / d}$. We see that for all $p=(V, k) \in P$ and $t \in[0,1], \tau(S(p, t))=t \tau_{p}$. Thus condition (i) as in the definition of an $\mathcal{L}$-set is satisfied. Now let $\rho \in(0,1)$ be arbitrary and let $\epsilon=\frac{1}{3}(1-\rho) \lambda$. Let $k \geq 0$ and $N_{k}=\left\{p \in P \mid \lambda^{k+1}<\tau_{p} \leq \lambda^{k}\right\}$. Let $X$ be any compact subset with diameter at most $\rho \lambda^{k+1}$. Let $Q$ be the set of all vectors $x=\frac{\mathbf{p}}{q}$ for which there exists $p=(V, k) \in P$ such that $x \in V, q \in M_{k}$ and $X \cap B\left(x, t q^{-(d+1) / d}\right) \neq \emptyset$ for some $t \in(0, \epsilon)$. Let $W$ be the smallest affine subspace of $\mathbb{R}^{d}$ containing $Q$. Then $W$ is a rational affine subspace and by Lemma 4.2 it is a proper affine subspace. Then $p^{\prime}=(W, k) \in N_{k}$ and the definition of $Q$ shows that $S(p, t) \cap X$ is contained in $S\left(p^{\prime}, t\right)$, for all $t \in(0, \epsilon)$. This shows that condition (ii) as in the definition of an $\mathcal{L}$-set is also satisfied. Therefore the set of badly approximable vectors is an $\mathcal{L}$-set.

Together with Corollary 3.7 this implies the following, which in particular, in view of Lemma 2.2, proves Theorem 1.5.

Theorem 4.3. Let $C$ be a compact subset of $\mathbb{R}^{d}$ admitting an $F F C\left\{\mathcal{C}_{n}\right\}$. For each $i \in \mathbb{N}$, let $A_{i}$ be an affine automorphism of $\mathbb{R}^{d}$. Let $B$ be the set of all badly approximable vectors in $\mathbb{R}^{d}$. Then $\bigcap_{i=1}^{\infty} A_{i}^{-1}(B) \cap C$ is a uniformly winning set with respect to $\left\{\mathcal{C}_{n}\right\}$, and hence in particular it is uncountable.

\section{Sets Associated With TORAL AUtomorphisms}

In this section we prove the uniformly winning property of certain subsets of $\mathbb{R}^{d}$ associated to automorphisms, and more generally surjective endomorphisms, of $\mathbb{T}^{d}$, $d \geq 1$, and deduce Theorem 1.6. The arguments involved extend those of the main theorem of [7].

As before, let $\mathbb{T}^{d}=\mathbb{R}^{d} / \mathbb{Z}^{d}, \pi: \mathbb{R}^{d} \rightarrow \mathbb{T}^{d}$ the quotient homomorphism. Also, for any surjective endomorphism $\rho$ of $\mathbb{T}^{d}$ let $C_{\rho}(x)=\overline{\left\{\rho^{i}(x) \mid i \in \mathbb{Z}^{+}\right\}}$for all $x \in \mathbb{T}^{d}$, and let $E_{\rho}=\left\{v \in \mathbb{R}^{d} \mid e \notin C_{\rho}(\pi(v))\right\}$, where $e$ denotes the identity element in $\mathbb{T}^{d}$.

Theorem 5.1. For any surjective endomorphism $\rho$ of $\mathbb{T}^{d}, E_{\rho}$ is an $\mathcal{L}$-set in $\mathbb{R}^{d}$.

Proof. Let $\rho$ be a surjective endomorphism of $\mathbb{T}^{d}$ and let $R \in G L(d, \mathbb{Q}) \cap M(d, \mathbb{Z})$ be the matrix inducing $\rho$, namely such that $\pi(R(v))=\rho(\pi(v))$ for all $v \in \mathbb{R}^{d}$. Suppose first that all its eigenvalues of $R$ are of absolute value at most 1 . As $R$ is an integral matrix this implies that all eigenvalues are all roots of unity, and there exists a proper $R$-invariant rational subspace $W$ of $\mathbb{R}^{d}$ such that the factor of $R$ on $\mathbb{R}^{d} / W$ is an automorphism of finite order. Then $W+\mathbb{Z}^{d} / \mathbb{Z}^{d}$ is a closed 
subgroup of $\mathbb{T}^{d}$, and all orbits of the factor of $\rho$ on $\mathbb{R}^{d} / W+\mathbb{Z}^{d}$ are finite. Hence $E_{\rho}$ contains the complement of $W+\mathbb{Z}^{d}$ of $\mathbb{R}^{d}$, which is an $\mathcal{L}$-set, by Remark 3.4. (iii) and (iv). Hence $E_{\rho}$ is an $\mathcal{L}$-set. We may therefore suppose that $R$ has an eigenvalue of absolute value greater than 1 .

Now for $t>0$, let $B_{t}=B(0, t)$ be the open ball in $\mathbb{R}^{d}$ with centre at 0 and radius $t$. Then

$$
E_{\rho}=\bigcup_{\delta>0}\left(\mathbb{R}^{d} \backslash \bigcup_{0}^{\infty} R^{-j}\left(\mathbb{Z}^{d}+B_{\delta}\right)\right)
$$

Let $\lambda \in(0,1)$ be given. Let $\mu$ be the maximum of the absolute values of the eigenvalues of $R$. Let $l \in \mathbb{N}$ be such that $\mu^{-l}<\lambda$. Let $c=|\operatorname{det} R|^{-l}$. Then $R^{-j}\left(\mathbb{Z}^{d}\right) \subseteq c \mathbb{Z}^{d}$, for $1 \leq j \leq l$. Also let $r>0$ be such that $R^{-j}\left(B_{1}\right) \subseteq B_{r}$ for all $j=0,1, \ldots, l$. For any nonnegative integer $k$ and $t>0$ we have

$$
\bigcup_{j=k l}^{(k+1) l-1} R^{-j}\left(\mathbb{Z}^{d}+B_{t}\right) \subseteq c R^{-k l}\left(\mathbb{Z}^{d}\right)+R^{-k l}\left(B_{t}\right)=R^{-k l}\left(c \mathbb{Z}^{d}+B_{r t}\right) .
$$

Now let $P=\mathbb{Z}^{d} \times \mathbb{Z}^{+}$and for any $p=(m, i) \in P, m \in \mathbb{Z}^{d}, i \in \mathbb{Z}^{+}$, and $t>0$ let

$$
S(p, t)=R^{-i l}\left(c m+B_{t}\right) .
$$

Then by the above observation $\bigcup_{\delta>0}\left(\mathbb{R}^{d} \backslash \bigcup_{p \in P} S(p, \delta)\right)$ is contained in $E_{\rho}$. We shall complete the proof by showing that conditions (i) and (ii) as in the definition of $\mathcal{L}$-sets are satisfied for this family $\{S(p, t)\}$.

There exists an $R$-invariant proper subspace $V$ of $\mathbb{R}^{d}$ such that $\mathbb{R}^{d} / V$ is of dimension 1 or 2 , and the factor action of $R$ on $\mathbb{R}^{d} / V$ is $\mu$ times an isometry. It follows therefore that

$$
\tau(S(p, t))=\tau\left(R^{-i l}\left(B_{t}\right)\right) \leq M t \mu^{-i l}
$$

for a suitable constant $M$ independent of $p$ and $t$. For all $p=(m, i), m \in \mathbb{Z}^{d}$, $i \in \mathbb{Z}^{+}$, let $\tau_{p}=M \mu^{-i l}$. Then by the preceding observation, condition (i) as in the definition of an $\mathcal{L}$-set holds.

Since all eigenvalues of $R^{-1}$ are of absolute value at least $\mu^{-1}$ it follows that there exists $\gamma>0$ such that $\left\|R^{-n} v\right\| \geq \gamma \mu^{n}\|v\|$ for all $v \in \mathbb{R}^{d}$ and $n \in \mathbb{Z}^{+}$. Then for any $t<c / 2, m \in \mathbb{Z}^{d}-(0)$ and $i \in \mathbb{Z}^{+}$, we have

$$
\begin{aligned}
d\left(c R^{-i l} m, R^{-i l}\left(B_{2 t}\right)\right) & \geq d\left(\mathbb{R}^{d}-R^{-i l}\left(B_{c}\right), R^{-i l}\left(B_{2 t}\right)\right) \\
& =d\left(\mathbb{R}^{d}-R^{-i l}\left(B_{c-2 t}, 0\right)\right) \geq \gamma(c-2 t) \mu^{-i l} .
\end{aligned}
$$

Now let $\rho \in(0,1)$ be such that $\rho<c \gamma /(3 M)$ and $\epsilon=c / 3$. For $k \in \mathbb{Z}^{+}$let $N_{k}=\left\{p \in P \mid \lambda^{k+1}<\tau_{p} \leq \lambda^{k}\right\}$. Suppose that $N_{k}$ is nonempty and let $p=(m, i)$ and $p^{\prime}=\left(m^{\prime}, i^{\prime}\right) \in N_{k}$. Then we have $\tau_{p}=M \mu^{-i l}, \tau_{p^{\prime}}=M \mu^{-i^{\prime} l}$, and since $\mu^{-l}<\lambda$ we get that $i^{\prime}=i$; namely, the $i$ component is the same for all $p$ in $N_{k}$. Also, for any $0<t<\epsilon=c / 3$, we have

$$
\begin{aligned}
d\left(S(p, t), S\left(p^{\prime}, t\right)\right) & =d\left(R^{-i l}\left(c m+B_{t}\right), R^{-i^{\prime} l}\left(c m^{\prime}+B_{t}\right)\right) \\
& =d\left(c R^{-i l}\left(m^{\prime}-m\right), R^{-i l}\left(B_{2 t}\right)\right) \\
& \geq \gamma(c-2 t) \mu^{-i l}=\gamma(c-2 t) \tau_{p} / M \\
& >(c \gamma /(3 M)) \lambda^{k+1}>\rho \lambda^{k+1}
\end{aligned}
$$


Therefore if $X$ is a compact subset with diameter at most $\rho \lambda^{k+1}$, then $X$ intersects $S(p, t)$ for at most one $p$ in $N_{k}$. This shows that condition (ii) in the definition holds and completes the proof of the theorem.

Corollary 5.2. Let $\mathbb{T}^{d}, d \geq 1$, and $\pi: \mathbb{R}^{d} \rightarrow \mathbb{T}^{d}$ be as above. Let $F$ be the subgroup of $\mathbb{T}^{d}$ consisting of all elements of finite order. Let $E$ be the subset of $\mathbb{R}^{d}$ consisting of $v \in \mathbb{R}^{d}$ such that for every surjective endomorphism $\rho$ of $\mathbb{T}^{d}, C_{\rho}(\pi(v))$ does not contain any element of $F$. Let $C$ be a compact subset of $\mathbb{R}^{d}$ with an $F F C\left\{\mathcal{C}_{n}\right\}$. Then $E \cap C$ is a uniformly winning set with respect to $\left\{\mathcal{C}_{n}\right\}$.

Proof. Since there are only countably many surjective endomorphisms, in view of Lemma 2.1 it suffices to show that for any surjective endomorphism $\rho$, the set of $v$ in $C$ such that $C_{\rho}(\pi(v))$ does not contain any element of $F$ is a uniformly winning set. Again, $F$ is countable, and hence by Lemma 2.1 it suffices to show that for any $f \in F,\left\{v \in C \mid f \notin C_{\rho}(\pi(v))\right\}$ is a uniformly winning set. Let $\rho$ be a surjective endomorphism and $f \in F$ be given. If $m$ is the order of $f$, then $\left\{v \in \mathbb{R}^{d} \mid f \notin C_{\rho}(\pi(v))\right\}$ contains $\left\{v \in \mathbb{R}^{d} \mid e \notin C_{\rho^{m}}(\pi(v))\right\}$, which by Theorem 5.1 is an $\mathcal{L}$-set. Thus $\left\{v \in \mathbb{R}^{d} \mid f \notin C_{\rho}(\pi(v))\right\}$ is an $\mathcal{L}$-set, and hence by Theorem 3.6 its intersection with $C$ is a uniformly winning set with respect to $\left\{\mathcal{C}_{n}\right\}$. This proves the corollary.

Theorem 1.6 now follows from Corollary 5.2 together with Lemma 2.2 .

\section{REFERENCES}

1. C.S. Aravinda and Enrico Leuzinger, Bounded geodesics in rank-1 locally symmetric spaces, Ergod. Th. Dynam. Sys. 15 (1995), 813-820. MR1356615 (96h:53050)

2. M. B. Bekka and M. Mayer, Ergodic theory and topological dynamics of group actions on homogeneous spaces, London Mathematical Society Lecture Note Series, 269. Cambridge University Press, Cambridge, 2000. MR.1781937 (2002c:37002)

3. R. Broderick, L. Fishman and D. Kleinbock, Schmidt's game, fractals, and orbits of toral endomorphisms, Ergod. Th. Dynam. Sys. 31 (2011), 1095-1117.

4. S.G. Dani, Bounded geodesics on manifolds of negative curvature, preprint, 1986 (unpublished).

5. S.G. Dani, Bounded orbits of flows on homogeneous spaces, Comment. Math. Helv. 61 (1986), 636-660. MR870710 (88i:22011)

6. S.G. Dani, On badly approximable numbers, Schmidt games and bounded orbits of flows, in Number Theory and Dynamical Systems, Eds.: M.M. Dodson and J.A.G. Vickers, Lond. Math. Soc. Lect. Notes 134, Cambridge University Press, 1989. MR.1043706 (91d:58200)

7. S.G. Dani, On orbits of endomorphisms of tori and the Schmidt game, Ergod. Th. and Dynam. Sys. 8 (1988), 523-529. MR980795 (90b:58145)

8. L. Fishman, Schmidt's game on fractals, Israel J. Math. 171 (2009), 77-92. MR 2520102 (2010d:11079)

9. L. Fishman, Schmidt's game, badly approximable matrices and fractals, J. Number Theory 129 (2009), 2133-2153. MR2528057 (2010j:11123)

10. D. Kleinbock and B. Weiss, Badly approximable vectors on fractals, Israel J. Math. 149 (2005), 137-170. MR2191212 (2008d:11079)

11. D. Kleinbock and B. Weiss, Modified Schmidt games and a conjecture of Margulis, preprint, 2010. arXiv:1001.5017vl [math.DS] 27 Jan 2010

12. C.T. McMullen, Winning sets, quasiconformal maps and Diophantine approximation, Geom. Funct. Anal. 20 (2010), 726-740. MR2720230 
13. W.M. Schmidt, On badly approximable numbers and certain games, Trans. Amer. Math. Soc. 123 (1966), 178-199. MR0195595 (33:3793)

14. W.M. Schmidt, Diophantine Approximation, Springer-Verlag, 1980. MR568710 (81j:10038)

School of Mathematics, Tata Institute of Fundamental Research, Homi Bhabha Road, Mumbai 400005, India

E-mail address: dani@math.tifr.res.in

Department of Mathematics, Indian Institute of Science, Bangalore 560012, India

E-mail address: hemangi@math.iisc.ernet.in 